\title{
Distance Distributions Measured from BSA to Norfloxacin Using Time-domain Fluorescence Resonance Energy Transfer
}

\author{
Min Liu \& Jian Shi \\ School of Physics and Electronic Engineering, Xuzhou Normal University \\ Xuzhou 221116, Jiangsu, China \\ E-mail: liuminxznu@qq.com \\ Yiman Liu \\ Department of Physics, Hunan University of Science and Technology \\ Xiangtan 411201, Hunan, China \\ E-mail: liuminxuzhouzhuzhou@126.com \\ Changde Peng (Corresponding author) \\ School of Physics and Electronic Engineering, Xuzhou Normal University \\ Xuzhou 221116, Jiangsu, China \\ Tel: 86-151-6225-7759Ｅ-mail: liuminxznu@126.com
}

Received: December 2, 2010 Accepted: January 19, 2011 doi:10.5539/apr.v3n1p128

This work was supported by the Natural Science Foundation of Hunan province (Nos. 09JJ3012) and Natural Science Foundation of Xu Zhou Normal University (Nos.09XL Y04).

\begin{abstract}
To measure the distance distribution of Bull Serum Albumin (BSA)-Norfloxacin pairs in solution, an improved numerical model in analyzing time-domain Fluorescence Resonance Energy Transfer (FRET) was developed. Compared to the classical model, the distance distribution function in the improved model is not necessary to be assumed. In the experiment, BSA-Norfloxacin pairs' distance distribution were investigated within the temperature ranging from $20 \sim 50{ }^{\circ} \mathrm{C}$ and BSA concentration ranging from $10^{-5}$ to $5 \times 10^{-5} \mathrm{~mol} / \mathrm{L}$. Experimental results analyzing by the improved model indicate: the typical distance of BSA-Norfloxacin pairs was at the range of $10^{-9} \sim 10^{-8} \mathrm{~m}$; the peak location of distance distribution function was $8.60 \mathrm{~nm}$ and half width was $12.40 \mathrm{~nm}$ at $20{ }^{\circ} \mathrm{C}, 1.0 \times 10^{-5} \mathrm{~mol} / \mathrm{L}$; the peak location and the half width of the distance distribution function could change regularly with the change of the temperature and the concentration.
\end{abstract}

Keywords: Fluorescence Spectrum, Fluorescence Resonance Energy Transfer, Time-domain

\section{Introduction}

An excited molecule can return to the ground state by transferring energy to another molecular and this process is called Fluorescence Resonance Energy Transfer (FRET) (Y. Liu, et al, 303. H. Hai, et al, 1053. M. madorin, et al, 1118). The original theory of FRET was firstly carried out by Förster. Regarding the latter, Gutierrez-Merino (C. G. Merino. 259 260), Fernandes and Ricardo C. Capeta (F. Femandes, et al, 334) derived some new models when they were studying crystal systems, polymer systems and membranes systems with lipid-protein (R. C. Capeta, et al,161 163). Due to the high sensitivity and a wide range of accessible timescales $\left(10^{-12} s \sim 10^{-8} s\right)$, FRET, a powerful tool to study conformational dynamics and spatial relationships between polymers and biopolymers, plays an especially particularly important role in biophysics and biochemistry researches. Recently, Eva Maria Galvez (E. M. Galvez, et al, 1367 1368) labeled the $\mathrm{H}^{+}$-ATP synthase with a hydrophilic quantum dot and an organic fluorescence acceptor and they investigated ensemble FRET and single-pair FRET. However, in their work, they didn't concern the distance distribution between donor and acceptor which we think is 
necessary for enzyme kinetics studies.

The rate of FRET is a function of the inverse in the sixth power of the distance between the energy donor and acceptor, which is used usually to probe distance and intermolecular dynamics (Peggy S. Eis et al, 23). In 1970s, Haas and coworkers (E. Hass, et al, 11 15, E. Hass, et al, 1807 1809) developed a method by which they studied distribution of end-to-end distances of oligopeptides in solution. In their method, the time-resolved decays data were used combined with FRET. Regarding the latter, a classical method of recovering distance distribution between donor and acceptor has been demonstrated by Joseph R. Lakowicz (J. R. Lakowicz, 1102 1105). However, in order to simulate the distance distributions, it's necessary to set a model in which the distance distributions between the donor and acceptor has been supposed (the classical model was Gaussian or Lorentzian). In fact, the reasons are far more enough to make us sure that the D-A pair distance distribution have to follow some models. In this work, an improved mathematical model has been reported, in which the D-A pair distance distribution can be recovered by solving a linear system rather than by prior assumption.

The interaction between small molecules (SM) and Bovine Serum Albumin (BSA) has attracted considerable attention in recent years (S. T. Kelly, et al, 972 973, Z. F. Cui, et al, 183 184, W. Norde, et al, 259 261, B, Yang, et al, 611 613). The importance of SM-BSA interactions has been demonstrated by Xin Yu-Jiang (X. Y. Jiang, et $a l, 1609)$ who analyzed the binding interactions of trans-resveratrol with BSA in dilute aqueous solutions in detail. In the present paper, we recovered the distance distribution between BSA (donor) and Norfloxacin (acceptor) using the improved model and analyzed the concentration effect and the temperature effect on this function.

\section{Materials and experimental methods}

\section{1 reagents and apparatus}

Bovine Serum Albumin (BSA), purity 98\%, was purchased from Shanghai Ruji Bio-tech Co., Ltd. Norfloxacin was obtained from Nanjing Pharmaceutical Factory Co., Ltd. All reagents were used without further purification. Tris- $\mathrm{HCl}$ buffer $\left(\mathrm{PH}=7.4\right.$, containing $\left.0.1 \mathrm{~mol} / \mathrm{L} \mathrm{Na}^{+}\right)$was prepared in order to control the $\mathrm{PH}$ value. All aqueous solutions were prepared using tri-distilled water. BSA solutions were diluted with Tris- $\mathrm{HCl}$ to obtain several $10-\mathrm{ml}$ samples and the concentrations of all of them were $1 \times 10^{-5} \mathrm{~mol} / \mathrm{L}$. Norfloxacin-BSA mixture solution which Norfloxacin concentration ranging from $0 \sim 5 \times 10^{-5} \mathrm{~mol} / \mathrm{L}$ were prepared.

The LIFETIME AND STEADY FLUORIMETER (FLS900) combined with fluorescence lifetime and steady-state spectrometer (C.Q. Han, et al, 309 310, Y, Liu, et al, 154, J. S. Kang, et al,143 145) manufactured from Edinburgh Instruments, Ltd., U.K., was used in our experiments. In the fluorescence lifetime spectrometer, the excitation source is a nanosecond flash lamp, $\mathrm{nF900,} \mathrm{engineered} \mathrm{with} \mathrm{a} \mathrm{fast} \mathrm{switching} \mathrm{thyratron,} \mathrm{spark} \mathrm{gap}$ and charging resistor. The flash lamp is capable of producing nanosecond and sub-second light pulses from 250 ns to $500 \mathrm{~ns}$ with repetition rates typically up to $50 \mathrm{KHz}$. The fluorescence lifetime spectrometer is based on the time correlated single photon counting (TCSPC) technique. The laboratory temperature was controlled at $20^{\circ} \mathrm{C}$.

\subsection{Fluorescence spectrum measurements}

The overlap integral $J(\lambda)$, expressing the degree of spectral overlap between the donor emission and the acceptor absorption, was an important factor which affected energy transfer rate. Figure 1 shows the overlap between BSA emission and Norfloxacin absorption. In the figure, the emission spectrum of BSA was measured with FLS900 ranging from $300 \sim 450 \mathrm{~nm}\left(\lambda_{e x}=280 \mathrm{~nm}\right)$ with setting a slit width of $2 \mathrm{~nm}$ for excitation and a slit width of $2.5 \mathrm{~nm}$ for emission. The absorption spectrum of Norfloxacin ranging from $300 \sim 380 \mathrm{~nm}$ was measured with a slit width of $4 \mathrm{~nm}$ for absorption. Time-resolved fluorescence spectrum's measurements were performed on the time-domain Fluorometer FLS900. The time-domain intensity decay of BSA was obtained at $350 \mathrm{~nm}$ $\left(\lambda_{E X}=280 \mathrm{~nm}\right)$ in a quartz cell with a slit width of $5 \mathrm{~nm}$ for excitation and an $8 \mathrm{~nm}$ bandwidth for emission in the absence and presence of Norfloxacin (figure 2).

\section{Theory analysis and result}

Fluorescence resonance energy transfer is the result of long rang dipole-dipole interaction (H. Szmacinski, et al, 185 187) between the donor and the accepter. For this interaction, it is necessary to have an overlap of the emission spectrum of the donor and the absorption spectrum of the acceptor. The more the overlap, the higher the efficiency of energy transfers. Figure 1 shows us that there is a large overlapped area between the emission spectrum of BSA and the absorption spectrum of Norfloxacin, which means the spectral properties of the fluorophores allow a FRET.

Figure 2 shows the time-domain intensity decay of BSA in the absence and presence of Norfloxacin. In figure 2, $\ln$ (counts) represents the logarithmic of fluorescence intensity. From figure2.b, it can be inferred that the BSA 
time-domain intensity decay in the presence of Norfloxacin is a multi-exponential shape which no longer to be a single-exponential shape liked figure2.a. The phenomenon means different BSA-Norfloxacin pairs display different decay times. In other words, the figure2.b implies that there was a distribution of distance between BSA and Norfloxacin. In section 3.1, we shall use the time-domain data to develop an improved numerical model for recovering the distance distribution $P(r)$.

\subsection{An improved model}

We know, the unique distance between a single donor and acceptor (R. S. Swathi, et al, 777) is given by

$$
k_{T}(r)=\frac{1}{\tau_{D}}\left(\frac{R_{0}}{r}\right)^{6}
$$

or

$$
k_{T}(r)=\frac{1}{\tau_{D}}\left(\frac{F_{D}-F_{D A}}{F_{D}}\right)
$$

where $k_{T}(r)$ is the D-A pair energy transfer rate, $r$ is the donor-to-acceptor distance, $R_{0}$ is the Förster distance at which FERT is $50 \%$ efficient, and $\tau_{D}$ is the decay time of the donor in the absence of acceptor, $F_{D}$ is the relative fluorescence intensity of the donor in the absence of acceptor while $F_{D A}$ is in the presence.

Assuming eq. 1 is correctly describes the transfer rate (S. Saini, et al, 23 24), the distance-dependent donor decay times is given by

$$
\frac{1}{\tau_{D A}}=\frac{1}{\tau_{D}}+k_{T}(r)
$$

where $\tau_{D A}$ is the decay time of the donor in the presence of acceptors. Recalling eq.3, the intensity decay of $\mathrm{D}-\mathrm{A}$ pairs spaced at a distance $r$ is given by

$$
I_{D A}(r, t)=I_{D}^{0} \exp \left(-t / \tau_{D A}\right)
$$

Where $I_{D}^{0}$ is the donor intensity at $t=0$. Eq. 3 and Eq.4 clearly demonstrate that the intensity decay follows a $r$ dependence where $r$ is the distance between the donor and the acceptor. So the intensity decay of the entire sample can be set up in the form:

$$
\begin{aligned}
& I_{D A}(t)=\int_{r=0}^{\infty} P(r) I_{D A}(r, t) d r \\
& =I_{D}^{0} \int_{r=0}^{\infty} P(r)\left[-\frac{t}{\tau_{D}}-\frac{t}{\tau_{D}}\left(\frac{R_{0}}{r}\right)^{6}\right] d r
\end{aligned}
$$

where $P(r)$ is a distance distribution function. It is widely assumed

$$
P(r)=\frac{1}{\sigma \sqrt{2 \pi}} \exp \left[-\frac{1}{2}\left(\frac{\bar{r}-r}{\sigma}\right)^{2}\right]
$$

In our scheme, we arbitrarily set $P(r)$ instead of setting $P(r)$ to be Gauss, Lorentzian or others. Figure 3 shows our scheme, consider $r \in\left[0, M R_{0}\right]$, and $r_{i}=r_{0}+i h$, where $R_{0}$ is Förster distance, $h=r_{i+1}-r_{i}$ is step. Assuming the possibility of donor at distance $r_{i}$ is $P\left(r_{i}\right)$. Assuming the measured instrument function is $L(t)$, and then calculated values of $I_{D A}^{C}$ for any times can be described by:

$$
I_{D A}^{C}\left(t_{j}\right)=\int_{k=1}^{j} L\left(t_{k}\right) I_{D A}\left(t_{j}-t_{k}\right) d t_{k}
$$

Using eq. (7) and above suppose, we have

with the understanding that

$$
\begin{aligned}
& I_{D_{A}}^{c}\left(t_{j}\right)=\sum_{k=1}^{j} L\left(t_{k}\right) \lim _{n \rightarrow \infty} \sum_{i=1}^{n} I_{D}^{0} P\left(r_{i}\right)\left[-\frac{\left(t_{j}-t_{k}\right)}{\tau_{D}}-\frac{\left(t_{j}-t_{k}\right)}{\tau_{D}}\left(\frac{R_{0}}{r_{i}}\right)^{6}\right]\left(r_{i+1}-r_{i}\right) \Delta t \\
& =I_{D}^{0} \sum_{k=1}^{j} \sum_{i=1}^{n \rightarrow \infty} L\left(t_{k}\right) \Delta t\left[-\frac{\left(t_{j}-t_{k}\right)}{\tau_{D}}\left(1+\frac{R_{0}^{6}}{r_{i}^{6}}\right) h P\left(r_{i}\right)\right.
\end{aligned}
$$

$$
S=\sum_{k=1}^{j} L\left(t_{k}\right) \Delta t\left[-\frac{\left(t_{j}-t_{k}\right)}{\tau_{D}}\left(1+\frac{R_{0}^{6}}{r_{i}^{6}}\right)\right] h
$$


and setting $I_{D A}^{m}=I_{D A}^{c}$, we obtain

$$
\frac{I_{D A}^{m}}{I_{D}^{0}}=\sum_{i=1}^{n} S \times P\left(r_{i}\right)
$$

Eq.11 is a liner system. Precisely, given $I_{D A}^{m}$, one can compute the distance distribution $P(r)$.

From the above description, we found that the distance-distribution analysis is moderately complex. The flowchart for distance-distribution analysis was given by figure 4, which may be easier to understand.

\section{2 results and discussion}

Using the model, the BSA -Norfloxacin distance were investigated. The distance distribution function $P(r)$ at temperature $20^{\circ} \mathrm{C}$ and concentration $1 \times 10^{-5} \mathrm{~mol} / \mathrm{L}$ was illustrated in figure 5 , indicating that the peak location of function is at $8.60 \mathrm{~nm}, h w=12.40 \mathrm{~nm}$. In our work, we always controlled $\mathrm{PH}=9.0$ because the peak of BSA fluorescence emission very sensitively dependent on PH value (L. Y. Xu, et al, 1615 1617).

It is critically important to consider the effect of temperature and concentration on distance distribution function $P(r)$. We studied the concentration effects at temperature $20{ }^{\circ} \mathrm{C}$. The $R_{0}$ value were calculated from spectral overlap shown in gray (figure 1) with assuming orientation factor value $\kappa^{2}=2 / 3$ and the quantum yield of the BSA in the absence of Norfloxacin $Q_{D}=0.312 \pm 0.002$. We considered interval $\left[0,2 \mathrm{R}_{0}\right]$ because of its low energy transfer efficiency at $r>2 R_{0} \quad(E<1.5 \%)$. We found, with the dilution of BSA concentration, there were no significant change in the shape of $P(r)$, but the peak of $P(r)$ become larger and $h w$ become wider (figure 6). The half width of $P(r)$ at $5 \times 10^{-5} \mathrm{~mol} / \mathrm{L}$ is $1.16 \mathrm{~nm}$ wider than at $1 \times 10^{-5} \mathrm{~mol} / \mathrm{L}$, and the peak of $P(r)$ become $9.68 \mathrm{~nm}$ from $8.60 \mathrm{~nm}$. In figure 6 , a method of fitting a curve to the measured data points so as to minimize the sum of the squares of the distance of the points from the curve has been used. The quadratic fitting line can also tell us the trend of peak position. The phenomenon of change can be interpreted that with the decrease of BSA concentration, the number of BSA molecules in certain volume will decrease. Therefore, the average distance between BSA and Norfloxacin will be changed, and the various possibilities of distance distribution will be increased accordingly, which lead to the peak position shift and the $h w$ changing.

We also studied the temperature effects at certain concentration (figure 7). The BSA concentration at $1 \times 10^{-5} \mathrm{~mol} / \mathrm{L}$, we studied the BSA-Norfloxacin pair distance distribution at temperature form $20^{\circ} \mathrm{C}$ to $50{ }^{\circ} \mathrm{C}$ (At high temperature or low, the structure of BSA will be transformed). The results indicated that the peak position of $P(r)$ become smaller and the $h w$ of $P(r)$ become wider with the temperature increasing. The half width of $P(r)$ at $50{ }^{\circ} \mathrm{C}$ is of $0.83 \mathrm{~nm}$ narrower than that at $20^{\circ} \mathrm{C}$, and the peak location of $P(r)$ become $8.93 \mathrm{~nm}$ from $8.60 \mathrm{~nm}$. In figure 7 , the quadratic fitting line can also tell us the trend of change. There were two factors, in our knowledge, bring the changes. 1: As protein's property, the BSA's activity will increased with the temperature turned to higher, which will make the possibilities of distance distribution increased accordingly.2: Brown motion will become faster with the temperature increasing, which will lead to peak location shift.

Certainly, all of above is just a rough relationship between conditions and $P(r)$, the precise quantitative law between them has not been found. Factually, we always just care the trend with conditions change rather than care the exact quantitative law in many cases.

\section{Conclusions}

In this report, an improved model on how to recover the distance distribution of D-A pairs from time-domain decay data is presented. Based on our model and EFRT theory, we analyzed the distance distribution between BSA and Norfloxacin. The result indicated that typical distance between BSA and Norfloxacin is $10^{-9} \mathrm{~m}$. As conditions change, the peak location and half width of distance distribution function also changed in small scales. The results will shed light into the interaction between small molecule and protein, and we hope the result is useful to monitor medicine-protein interactions in living cell.

\section{References}

B. Yang, C. E. Wyman. (2006). BSA treatment to enhance enzymatic hydrolysis of cellulose in lignin containing substrates, Biotechnology and Bioengineering, 94, 611.

C. G. Merino. (1981). Quantitation of the Föster energy transfer for two-dimensional systems. II. protein distribution and aggregation in biological, Bio. Chem. 14, 259.

C. Q. Han, Y. Liu, Y. Yang, X. W. Ni, J. Lu, \& X. S. Luo. (2010). Study on fluorescence spectra of molecular association of acetic acid-water, Chin. Opt. Lett. 8, 309.

E, Haas, E. K. Katzir, \& L. Z. Steinberg. (1978). Brownian motion of the ends of oligopeptide chains in solution 
as estimated by energy transfer between the chain ends, Biopolymers, 17, 11.

E. Haas, M. Wilchek, E. K. Katzir, \& I. Z. Steinberg. (1975). Distribution of end-to-end distances of oligopeptides in solution as estimated by energy transfer, Proc. Natl. Acad. Sci. 72, 1807.

E. M. Galvez, B. Zimmermann, V. R. Riegraf, R. Bienert, \& P. Gräber. (2008). Fluorescence resonance energy transfer in single enzyme molecules with a quantum dot as donor, Eur. Bio. J., 37, 1367.

F. Fernandes, L. M. S. Loura, R. Koehorst, R. B. Spruijt, M. A. Hemminga, A. Fedorov, M. prieto. (2004). Quantification of protein-lipid selectivity using FRET: Application to the M13 major coat protein. Biophys. J., $87,344$.

H. Hai, T. Miura, T. Kobayashi, Y. Maeda, \& M. Miki. (2002). Conformational changes of the Troponin-Tropomyosin complex on F-Actin observed by fluorescence resonance energy transfer measurements, Journal of Fluorescence, 10, 1053.

H. Szmacinski, W. Wiczk, M. N. Fishman, P. S. Eis, J. R. Lakowicz, \& M. L. Johnson. (1996). Distance distributions from the tyrosyl to disulfide residues in the oxytocin and [Arg8 ]-casopressin measured using frequency-domain fluorescence resonance energy transfer, Eur. Biophys. J., 24, 185.

J. R. Lakowicz. (2008). Principles of Fluorescence Spectroscopy (Photocopy ed), Beijing: science press, (chapter 16).

J. S. Kang, J. R. Lakowicz, \& G. Piszczek. (2005). DNA dynamics: a fluorescence resonance energy transfer study using a long-lifetime metal-ligand complex, Arch. Pharm. Res, 25,143.

L. Y. Xu, \& W. Q. Yu. (2004). The fluorescence characteristics of Norfloxacin and its application, Spectroscopy and Spectral Analysis, 12, 1615.

M. madörin, P. V. Hoogevest, R. Hilfiker, \& H. Leuenberger. (2000). The use of Fluorescence resonance transfer to study the disintegration kinetics of liposomes containing lysolecithin and oleic acid in rat plasma, Pharmaceutical Research, 17,1118.

P. S. Eis, J. Kušba, M. L. Johnson, \&J. R. Lakowicz. (1993). Distance distributions and dynamics of zinc finger peptide from fluorescence resonance energy transfer measurements, Journal of Fluorescence, 3, 23.

R. C. Capeta, J. A. Poveda, \& L. M. S. Loura. (2006). Non-uniform membrane probe distribution in resonance energy transfer: application to protein-lipid selectivity, Journal of Fluorescence, 16, 161.

R. S. Swathi, \& K. L. (2009). Sebastian, Distance dependence of fluorescence resonance energy transfer, Ind. Aca. Sci., 121, 777.

S. Saini, H. Singh, B. Bagchi. (2006). Fluorescence resonance energy transfer (FRET) in chemistry and biology: non-Föster distance dependence of the FRET rate, Indian. Aca. Sci, 11823.

S. T. Kelly, \& A. L. Zydney. (2004). Effects of intermolecular thiol-Disulfide interchange reactions on BSA fouling during microfiltration, 44, 972 .

W. Norde, \& C. E. Giacomelli, (2000). BSA structural changes during homomolecular exchange between the adsorbed and the dissolved states, J. Biotechnology, 79, 259.

X. Y. Jiang, W. X. Li, \& H. Cao. (2008). Study of the interaction between trans-resveratrol and BSA by the multi-spectroscopic method, J. Solution. Chem. 37, 1609.

Y. Liu, H. Shao, X. W. Ni, \& J. Lu. (2008). Formation mechanism of ethanol-water excimer, Chin. Opt. Lett, 6, 154.

Y. Liu, X. S. Luo, Z. H. Shen, J. Lu, \& X.W. Ni. (2006). Studies on molecular structure of ethanol-water clusters by fluorescence spectroscopy, Opt. Rev., 13, 303.

Z. F. Cui, \& K. I. T. Wright. (1994). Gas-liquid two-phase cross-flow ultrafiltration of BSA and dextran solutions, J. Membrane. Sci., 90, 183. 


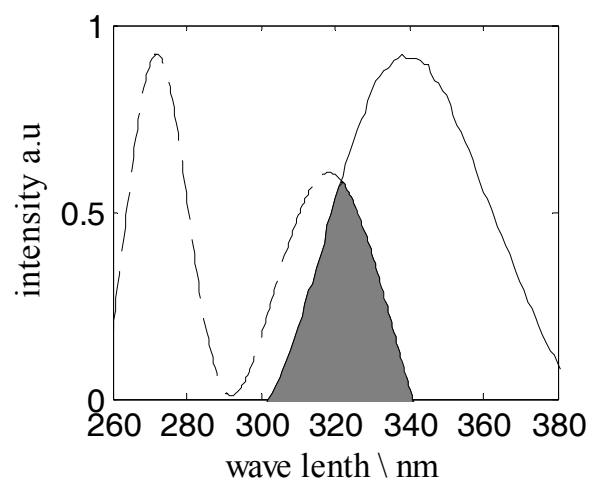

Figure 1. Absorption of Norfloxacin and Emission of BSA. Solid: the emission spectrum of BSA. Dash: the absorption of Norfloxacin. Grey area: the overlap between BSA emission and Norfloxacin absorption
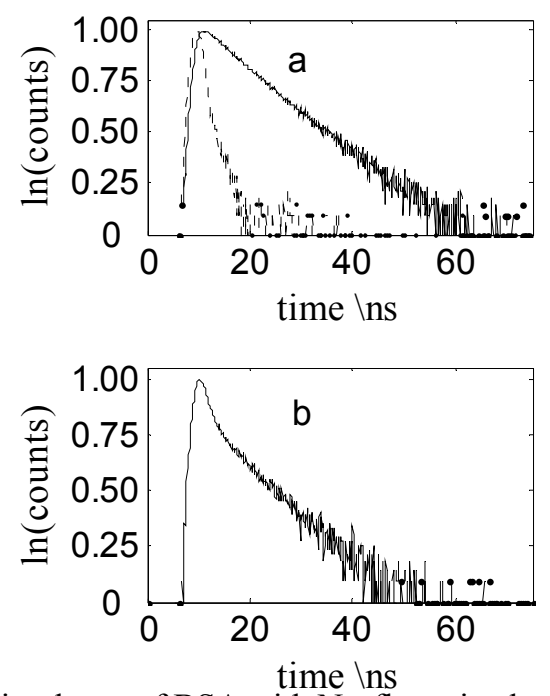

Figure 2. Time-domain intensity decay of BSA with Norfloxacin absent (a) and present (b). Dash in (a): The instrument response function. Solid: The decay of the logarithmic of fluorescence intensity. (Sometimes, the solid line in the figure looks like black dot because the measure data is a discrete form.)

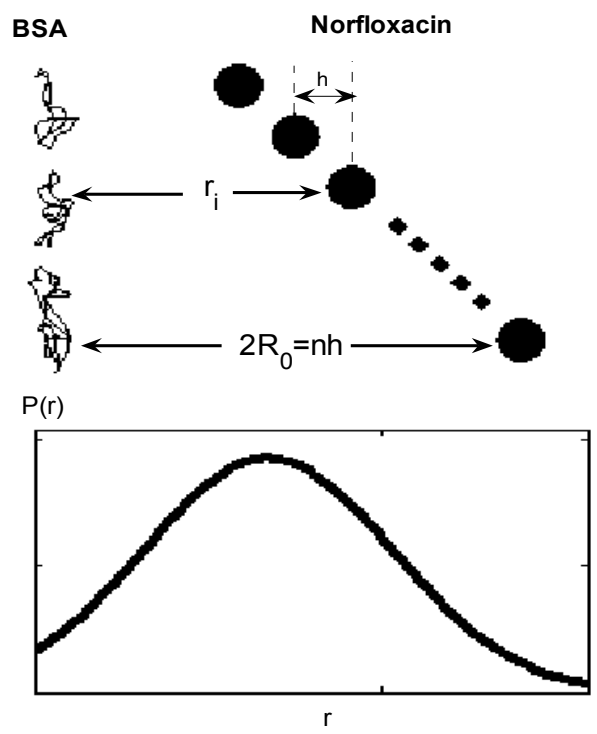

Figure 3. Distance distribution function P(r) in improved model 


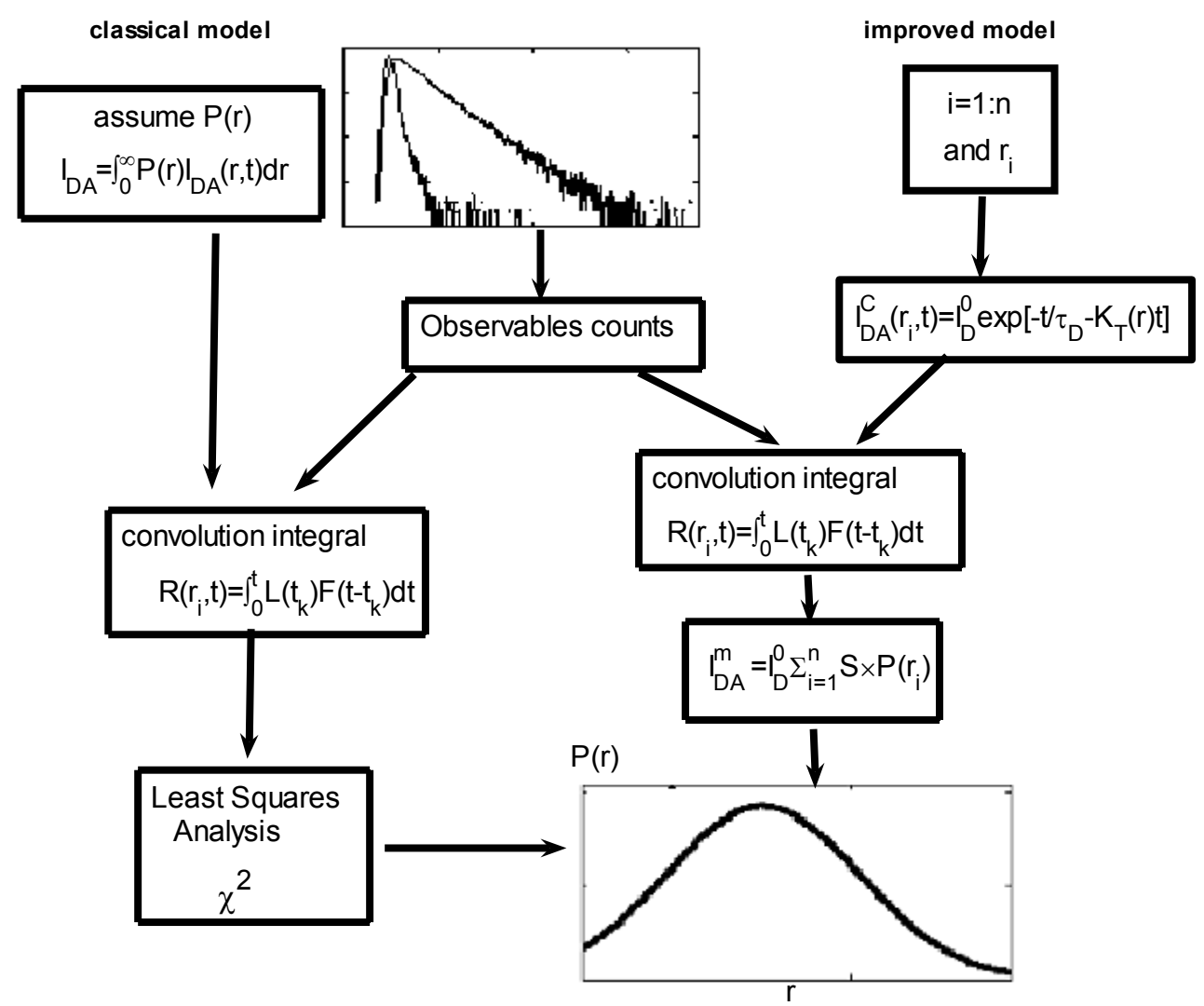

Figure 4. Flowchart for distance distribution analysis of classical model and improved model

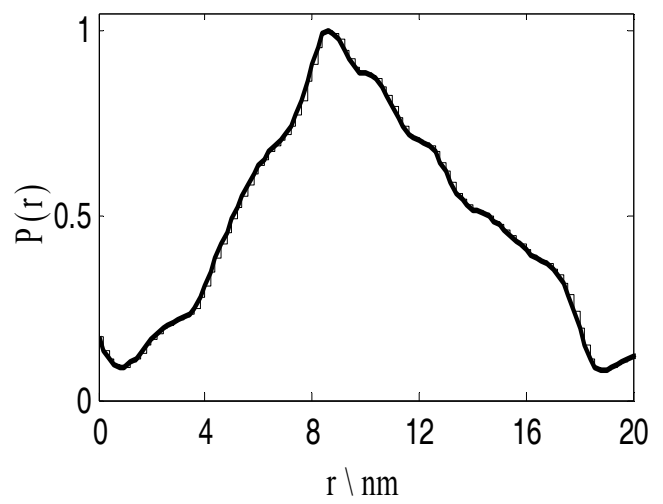

Figure 5. Distance distribution between BSA and Norfloxacin at $20{ }^{\circ} \mathrm{C}, \mathrm{PH}=9 \mathrm{C}_{\mathrm{BSA}}=10^{-5} \mathrm{~mol} / \mathrm{L}$. Dash: the distance distribution recovered by improved model. Solid: the envelope curve of distance distribution functions 

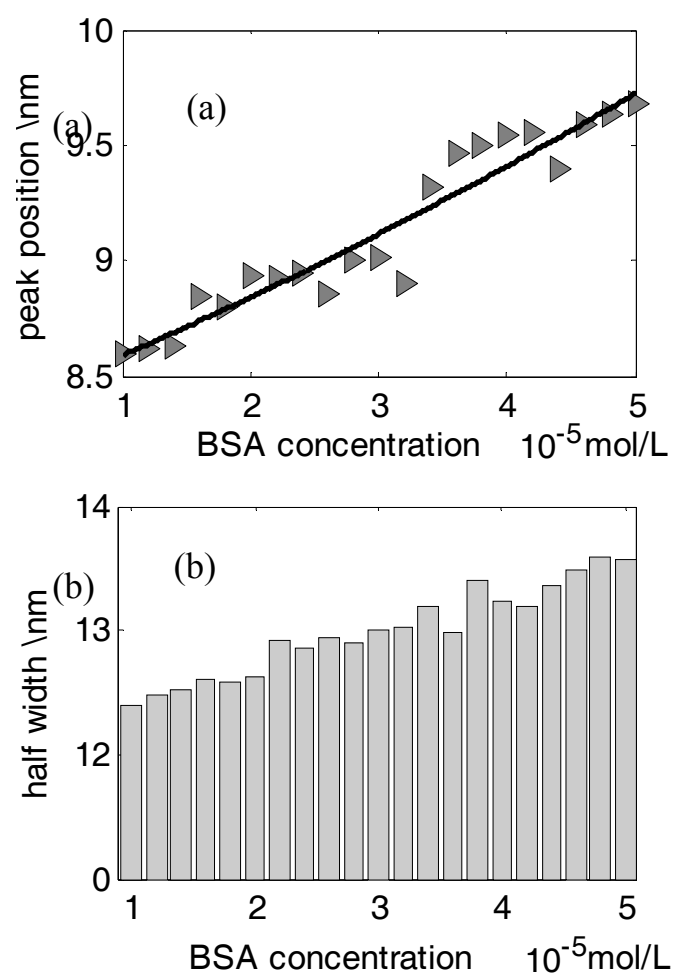

Figure 6. The concentration effects on the peak position and the half width of $\mathrm{P}(\mathrm{r})$. Black line in (a): the quadratic fitting curve of the data points
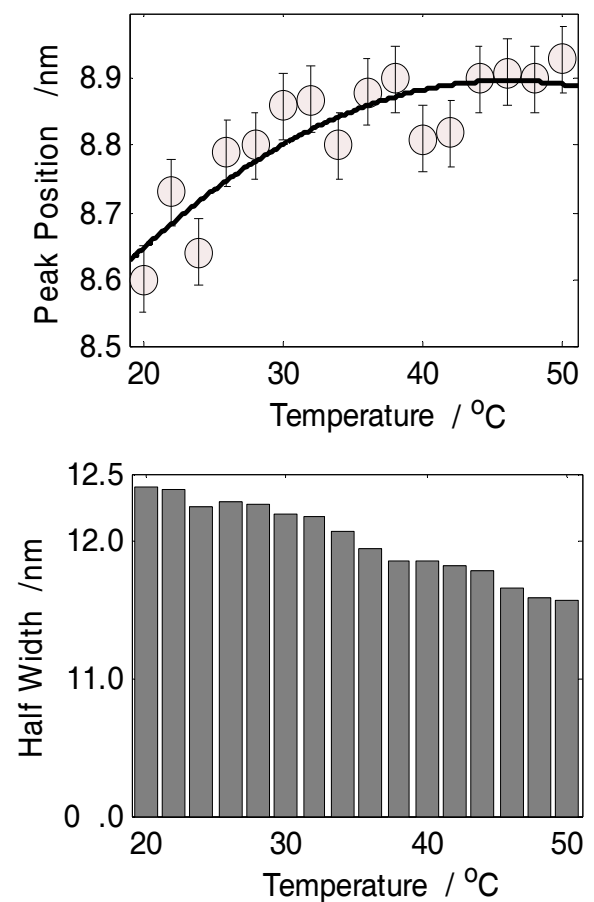

Figure 7. The temperature effects on the peak position and the half width of $\mathrm{P}(\mathrm{r})$. Black line in (a): the quadratic fitting curve of the data points 